\title{
A relationship between apparent extent and alignment in the Poggendorff illusion
}

\author{
SUZANNE GREIST-BOUSQUET \\ Kean College of New Jersey, Union, New Jersey \\ H. R. SCHIFFMAN and RICHARD DORSETT \\ Rutgers-The State University, New Brunswick, New Jersey \\ and \\ JANET DAVIS \\ Drew University, Madison, New Jersey
}

\begin{abstract}
Based upon the suggestion that apparent misalignment of transversals in the Poggendorff illusion is attributable to an underestimation of the linear extent between the parallels, 50 subjects made alignment and extent estimates in a diagonally oriented variation of the Poggendorff figure. Previous findings of significant misalignment and underestimation were verified, and a significant correlation was found between alignment judgments and extent judgments. Thus, additional evidence was provided for the relationship between misalignment and underestimation in the Poggendorff illusion.
\end{abstract}

In the classic Poggendorff illusion (Figure 1A), the two collinear oblique lines, or transversals, appear to be noncollinear or misaligned. Several investigators (e.g., Pressey, 1971; Quina-Holland, 1977; Zanuttini, 1976) have suggested that this misalignment illusion is due to an underestimation of the distance between the parallels. Figure 1 illustrates how underestimation of interior extent may promote a misalignment effect.

Following much earlier work (Judd, 1899), GreistBousquet and Schiffman (1981a) verified empirically the intertransversal underestimation, and suggested that the underestimation is due to the influence of an amputated form of the wings-in Müller-Lyer figure contained within the Poggendorff parallels (Figure 2B). Other investigators have also found underestimation of inner oblique extent when subjects produce (Weintraub \& Tong, 1974), adjust (Judd, 1899), or compare (Quina-Holland, 1977) stimuli that are equivalent in content and orientation (i.e., an unfilled oblique extent). Although an analogous wingsout figure is also present in the Poggendorff parallels (Figure 2C), previous research on the Müller-Lyer illusion (e.g., Greist-Bousquet \& Schiffman, 1981b; Warren \& Bashford, 1977) indicates that this amputated variation produces little overestimation.

If misalignment in the Poggendorff illusion is attributable to an underestimation of the intertransversal extent, then one would expect judgments of intertransversal extent and transversal alignment to be correlated. In the experiment reported here, we examined whether alignment

This research was supported by a grant from the Research Council of Rutgers-The State University to H. R. Schiffman. Correspondence may be addressed to Suzanne Greist-Bousquet, Department of Psychology, Kean College of New Jersey, Union, NJ 07083. and extent estimates are correlated within the same diagonally oriented variation of the Poggendorff illusion.

\section{METHOD}

\section{Subjects}

Fifty undergraduate Rutgers University students served as subjects. All had corrected or uncorrected visual acuity of 20-20.

\section{Stimuli}

Diagonally oriented variations of the Poggendorff illusion (Figure 3A) were prepared with black graphic tape $(0.4 \mathrm{~mm}$ wide) on clear acetate and mounted on white posterboard $(215 \times 280 \mathrm{~mm})$. Poggendorff figures were constructed with $45^{\circ}$ oblique parallels that were $80 \mathrm{~mm}$ long and $15 \mathrm{~mm}$ apart at right angles to their length. The vertical extent between the transversals was $22 \mathrm{~mm}$. The transversal segments were $10 \mathrm{~mm}$ long.

Control figures were also constructed. The extent-control figure consisted of two dots (Figure 3B) that were $1.5 \mathrm{~mm}$ in diameter; the alignment-control figure consisted of two transversals (Figure 3C).

For figures in which alignment was to be estimated, the upper transversal was mounted separately and was adjustable. The lower part of the figure (containing either two parallels and a single vertical transversal or the single vertical transversal alone) was stationary. In the Pog-

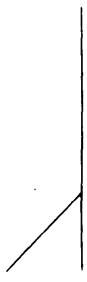

A

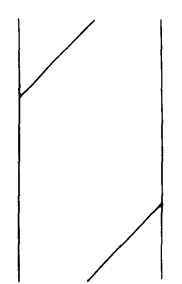

B

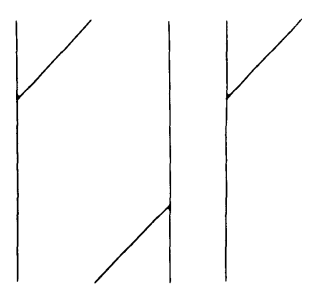

C
Figure 1. The classic Poggendorff illusion (A) may be attributable to an underestimation of the distance between the parallels. By moving the right parallel laterally, an increase in interior extent produces the impression of subjective collinearity $(B)$, whereas a decrease in interior extent accentuates the misalignment effect (C). 

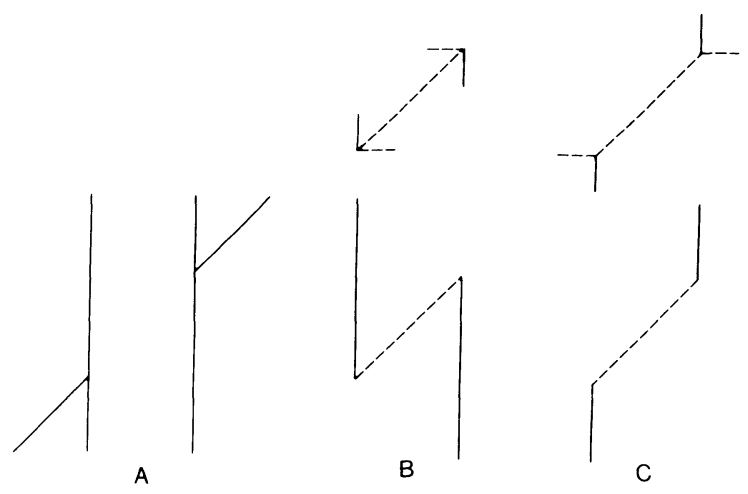

Figure 2. The Poggendorff figure (A) contains an amputated version of the wings-in Müller-Lyer illusion (B) and an amputated version of the wings-out Müller-Lyer illusion (C). (The complete MüllerLyer figure is shown above $B$ and $C$.)

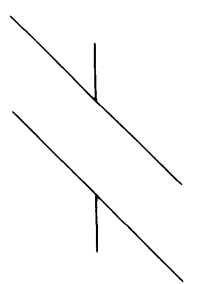

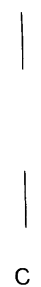

Figure 3. Diagonally oriented Poggendorff and control figures used in the present experiment. Subjects adjusted the upper transversal in A so that it appeared collinear with the lower transversal and recreated the extent between the transversals in A. Subjects recreated the extent in a two-dot control figure (B) and adjusted the upper transversal to apparent collinearity with the lower transversal in a second control figure (C).

gendorff figure, the upper transversal was movable along the top diagonally oriented parallel line. In the control figure, the upper transversal was movable along an equivalent extent in a figure without the parallels.

For figures in which extent was to be estimated, the Poggendorff figure was centered in the right half of the posterboard. A single dot was centered in the empty field to the right of the Poggendorff and control figures.

\section{Procedure}

For estimates of alignment, the subjects were asked to adjust the upper transversal so that it appeared aligned or collinear with the lower transversal segment. This was accomplished by sliding the top half of the figure, which contained the single transversal, along the edge of the lower half of the figure. For estimates of intertransversal extent, the subjects were asked to recreate the extent to the right of the stimulus figure. A single dot was provided in the center of the right half of the stimulus, and the subjects placed a dot above it such that the distance between the two dots appeared equivalent to the intertransversal or interdot extent presented in the figure. Each subject made four judgments (two alignment and two extent judgments) and the presentation order of stimuli was randomized for each subject. Subjects were tested individually.

\section{RESULTS}

Estimates of extent were measured to the nearest $0.5 \mathrm{~mm}$. The difference between the extent produced by the subject and the true intertransversal extent was recorded; underestimations were scored as negative. Estimates of alignment were also measured to the nearest $0.5 \mathrm{~mm}$. The horizontal difference between the point of apparent alignment produced by the subject and exact vertical alignment was recorded. Errors in the typical direction of the Poggendorff illusion (i.e., adjusting the upper transversal to the right of the true extension of the lower transversal) were scored as negative.

The mean extent judgment for the two-dot control figure was $-0.44 \mathrm{~mm}(S D=2.56)$. This was not significantly different from zero $[t(49)=-1.21, p>.05]$ and, therefore, judgments of the control extent were not subtracted from the intertransversal judgments for the Poggendorff figure. The mean extent judgment for the Poggendorff figure was $-1.56 \mathrm{~mm}(S D=2.60)$; this was significantly different from zero $[t(49)=-4.25, p<.0001]$. This result (i.e., underestimation) is consistent with previous findings of underestimation for conventionally oriented Poggendorff figures (Greist-Bousquet \& Schiffman, 1981a).

The mean alignment judgment for the Poggendorff figure was $-0.88 \mathrm{~mm}(S D=1.27)$ and was significantly different from zero $[t(49)=-4.89, p<.0001]$. The mean alignment judgment for the control figure was $-0.64 \mathrm{~mm}(S D=1.12)$ and also was significantly different from zero $[t(49)=-4.04, p<.0002]$. Therefore, control alignment judgments were individually subtracted from Poggendorff alignment judgments. A Pearson moment correlation coefficient was derived for Poggendorff alignment estimates and Poggendorff extent estimates and was significant $(r=.234, p<.05)$. Thus, intertransversal extent judgments were positively correlated with alignment judgments for the same Poggendorff figure.

\section{DISCUSSION}

Historically, the Poggendorff and Müller-Lyer illusions have been considered separately, each with its own distortion-producing mechanism. The present findings document a relationship between the two illusion effects and, together with previous findings (Greist-Bousquet \& Schiffman, 1981a, 1985), suggest that the Poggendorff and MüllerLyer figures, in part, share a common basis. We have not argued that the two illusions produce identical distortion effects. In the classic presentation of the Müller-Lyer illusion, there is an obvious distortion of linear extent when one compares wings-in and wings-out figures. In contrast, the less obvious distortion of intertransversal extent in the Poggendorff figure promotes the apparent noncollinearity of the transversals. Since controversy and confusion continue in explorations of visual illusions, it is important to identify the commonalities among illusion effects.

\section{REFERENCES}

Greist-Bousquet, S., \& Schiffman, H. R. (1981a). The Poggendorff illusion: An illusion of linear extent? Perception, 10, 155-164.

GreisT-BousQueT, S., \& SCHIFFMAN, H. R. (1981b). The role of structural components in the Mueller-Lyer illusion. Perception \& Psychophysics, 30, 505-511.

Greist-Bousquet, S., \& SChiffman, H. R. (1985). Poggendorff and Müller-Lyer illusions: Common effects? Perception, 14, 427-447. Judd, C. H. (1899). A study of geometrical illusions. Psychological Review, 6, 241-261. 
Pressey, A. W. (1971). An extension of assimilation theory to illusions of size, area, and direction. Perception \& Psychophysics, 9 , 172-176.

Quina-Holland, K. (1977). Spatial distortions within the Poggendorff figure and its variants: A parametric analysis. Perception \& Psychophysics, 21, 118-124.

WARREN, R. M., \& BASHFord, J. A. (1977). Müller-Lyer illusions: Their origin in processes facilitating object recognition. Perception, 6, 615-626.
Weintraub, D. J., \& Tong, L. (1974). Assessing Poggendorff effects via collinearity, perpendicularity, parallelism, and Oppel (distance) experiments. Perception \& Psychophysics, 16, 213-221.

ZanutTini, L. (1976). A new explanation for the Poggendorff illusion. Perception \& Psychophysics, 20, 29-32.

(Manuscript received May 12, 1989.) 